\title{
The Visual Dipole - A Key to Understanding Antenna Theory
}

\author{
Paul Crilly \\ Department of Engineering, Electrical Engineering \\ U.S. Coast Guard Academy, New London, CT \\ Paul B. Crilly@uscga.edu
}

\begin{abstract}
We present a simple, yet elegant pedagogical approach to the study of antenna theory that provides a sensory experience by using the resonant half-wave dipole as the starting point. This approach enables the student to more easily apply this basic knowledge to understand other antenna types. We start with the resonant half-wave dipole that when energized produces a periodic current and voltage standing wave $(S W)$ along the wire. The two are out of phase such that at the center feed point, the current is a maximum and voltage is a minimum and thereby the transmission line sees a pure resistance of 73 ohms. To illustrate the current standing wave, we divide the two quarter wave sections into several pieces then series connected using incandescent light bulbs. We then energize the dipole, causing the bulbs to light where the brightness varies as a function of the current magnitude, the ones closest to the feed point have maximum brightness, the bulbs toward the ends less so. Hence the students are given a visual experience of the current $S W$ profile. This is extremely profound since the students see that there is indeed a displacement current which causes the bulbs to be illuminated even though the dipole ends are not terminated. This also conveys to them that circuit theory is not adequate to explain radio frequency phenomena (i.e. current only flows through a load if there is a return path). From this, the student can readily grasp why the feed impedance is 73 ohms, why the radiation pattern is donut shaped, why the dipoles can work at multiple bands and the operation of other SW antennas. Preliminary assessments show this approach has improved retention of antenna concepts.
\end{abstract}

\section{Keywords: Antennas, dipoles, antenna pedagogy, standing wave antennas, antenna education}

\section{Introduction}

The resonant half wave dipole is the basis of most other antennas. Understand it, and you can quickly grasp the operation of other antenna types such as the vertical, long wire, and Yagi.

Many antenna courses or textbooks start out with the Hertizian dipole, and/or the waveguide horn, and eventually cover the half-wave dipole [1]-[4]. However, for most students, operation of standing wave antennas such as the resonant dipole is quite mysterious. In fact, many do not understand the difference between it and its traveling wave counterpart. Students entering an antennas course already have a background in circuit theory and thus some have difficulty grasping the concept of displacement current and of how a dipole, an open circuit at the end of a transmission line, can carry a current and radiate an electromagnetic wave - a standing wave radiator. After all, they have learned in order for a generator to create a current flow, the load must be a closed circuit. Although they learn the theory, at least well enough to pass the course, many may still they have difficulty visualizing its operation and therefore, may not develop the necessary insight into its operation, its radiation pattern, how it can be used on other bands, why its feed impedance is a relatively low 73 ohms, and then how to extrapolate this knowledge to understand other types of standing wave antennas, or reengineer the dipole so it can work on other bands. For many, this knowledge becomes a matter of faith in their textbook and instructor, and/or a matter of empirical experience. For some antenna practitioners, the latter may be especially problematic because as they will be limited in their engineering practice to solutions that are extensions of existing designs.

In subsequent sections of this paper, we present an alternative approach to augment the teaching of antenna theory that is experiential and reinforces the necessary theory of antenna design. These will be used in the lecture and laboratory portions of the course.

\section{Dipole Basics}

We start with the half-wave $(\lambda / 2)$ center fed dipole as shown in Figure 1 with the indicated voltage and current standing wave profile. Note that at the center, the current is a maximum and voltage is a minimum and vice versa for the end points thus giving us $Z_{\text {feed }}=V_{\min } / I_{\max }=73 \Omega$. If we move the transmission line feed point away from the center, we get higher feed impedance, such that an end fed dipole would have $Z_{\text {feed }} \approx 3000-5000 \Omega[5]$. 


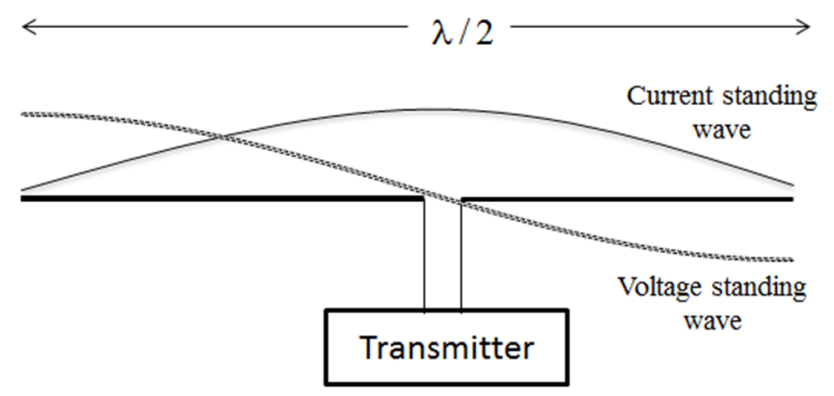

Figure 1. $\lambda / 2$ dipole showing the standing wave voltage and current profiles.

To demonstrate the current standing wave profile, we break up the antenna into sections and connect these using incandescent lights. This is shown in Figure 2. When the dipole is energized, the bulbs light up, with their intensity a function of the current profile, maximal bright at the center and less so towards the ends. In contrast with a terminated traveling wave antenna, there is no standing wave, and thus the bulbs would all have the same intensity. This demonstration reinforces the standing wave current/voltage nature of the dipole. The current profile also suggests a single lobed radiation pattern parallel to its length.

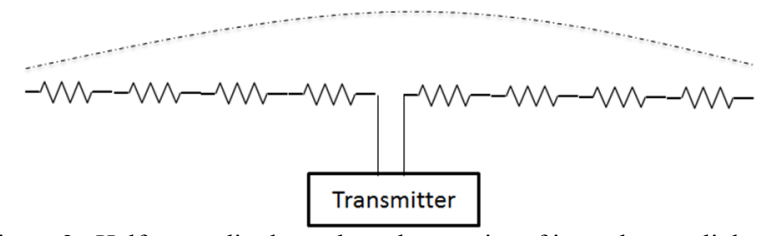

Figure 2. Half-wave dipole made up by a series of incandescent light bulbs shown with current standing wave profile shown in dotted plot.

\section{Multiband Dipoles}

We next consider using the $\lambda / 2$ dipole on multiple bands. Students are taught that the standing wave is periodic, and thus if the frequency is such that the dipole's length is $l=3 \lambda / 2$, we get the voltage/current profile shown in Figure 3. If you carefully observe Figure 3 , you will note that like the $\lambda / 2$ case, current and voltage magnitude at the feed point are the same as that of the $\lambda / 2$ case thus the feed impedance is 73 ohms. In fact, this is the case for all odd multiples of $\lambda / 2$. Thus our dipole can serve as a multiband radiator. Furthermore, the cyclic nature of the current profile suggests that the radiation pattern will also have multiple lobes and thus increased directionality and gain.

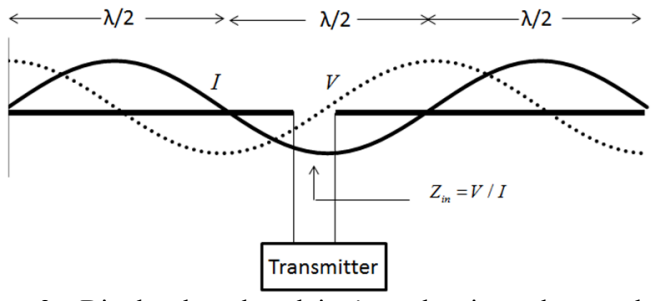

Figure 3. Dipole whose length is $3 \lambda / 2$ showing voltage and current profiles.

To reinforce the concept that our dipole will have a relatively low SWR at odd multiples of half wavelengths, we have the students construct a $\lambda / 2$ dipole for $7 \mathrm{MHz}$, and then measure the SWR from $7 \mathrm{MHz}$ to $150 \mathrm{MHz}$ using the Keysight $^{\mathrm{TM}} 9912 \mathrm{RF}$ analyzer. As Figure 4 shows, frequencies at odd multiples of $7 \mathrm{MHz}$, have a voltage standing wave ratio (VSWR) below 2. At all other frequencies, the VSWR is considerably above 2 .

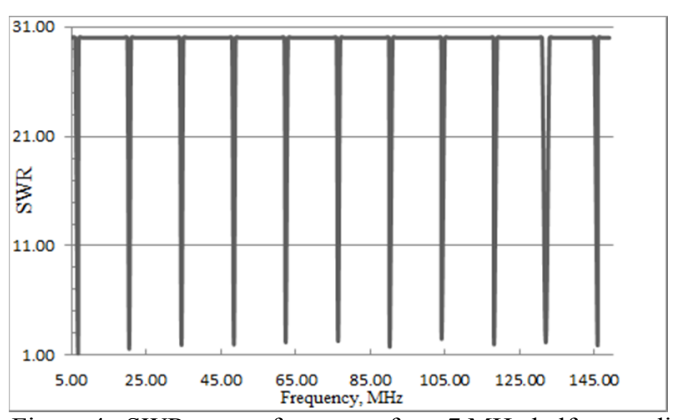

Figure 4. SWR versus frequency for a $7 \mathrm{MHz}$ half-wave dipole.

We then have the students get precise frequency values where the SWR is a minimum and have them note that the lowest points are not exactly at integer multiples of the fundamental frequency. The question is posed, why not? Previously, they assumed that a standing wave is a periodic sinusoidal shape. But this experiment forces them to reconsider this assumption. After some investigation and reading the literature, they learn that along the dipole, the current must physically reach zero at the end points and thus the SW shape may be distorted. In fact, regardless of length, the current always goes to zero at the end points. Thus if the dipole is significantly shorter than an electrical half wavelength, there is a relatively large deviation from the expected sinusoidal shape. Another reason for current wave distortion is electromagnetic self-coupling, or coupling with other nearby conductors (e.g. the ground).

We then pose the question, how can we design a multiband dipole that will work at other than odd multiples of its resonant frequency? After examination of Figure 3, the students discover that if the feed point is shifted off center 
might result in an acceptable SWR for the frequency bands of interest.

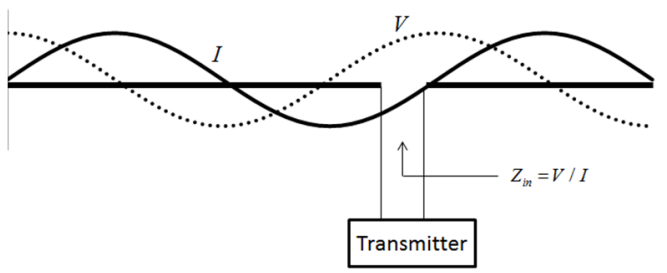

Figure 5. Off center dipole to enable multiband operation.

This is shown in Figure 5. They can visualize the voltage/current ratio changing as the feed point moves from the center to the edge, and that this ratio is a function of input frequency and thus they deduce why the feed impedance may have a reactive component as well as not be a $50-70 \mathrm{ohm}$ impedance, and thus they begin to appreciate why a matching network may be required in order to obtain the minimum SWR.

Later in the dipole discussion, the students learn that a $\lambda$ dipole, in other words, a $\lambda / 2$ dipole being used at its second harmonic has a beam width of $47^{0}$ versus a beam width of $78^{\circ}$ of its half-wave counterpart [1]. However, the full wave antenna has a relatively high SWR. Again, we ask why? Again students examine the current/voltage profile for a full wave dipole, and thereby note that when fed at the center, the feed impedance is nearly the same as the end fed $\lambda / 2$ dipole. Hence, without a matching network, the relatively high feed impedance would present a large mismatch for a $50 \mathrm{ohm}$ coaxial line.

Finally, they observe that when measuring the SWR at frequencies where the dipole's electrical length is extremely long relative to its lowest resonant frequency, the dipole starts to act like a traveling wave antenna. Again, the question is posed why? The answer being that at long lengths, the magnitude of the reflected wave is greatly reduced and therefore, the dipole is no longer a standing wave antenna.

\section{Assessment and Conclusions}

We have presented an introduction to antenna theory that begins with the half-wave resonant dipole. The light bulb class demo provides the students a visual experience where students can see the current profile along the antenna's length and thereby can visualize the current distribution and thus develop a sense for its corresponding radiation pattern. By visualizing the current and voltage standing wave profile, they develop a better intuitive sense and thereby figure out what frequencies a given wire antenna will be suitable for, the ones that require a matching network. They also see that an antenna has physically real currents and voltages along its length which has safety implications if they should decide to use a loading coil, capacitive hat, or traps to electrically alter the antenna's length. Measuring the antenna's SWR over a wide range of frequencies further reinforces the theory of the periodicity of current and voltage standing waves. Preliminary assessment indicates that this approach greatly enhances understanding the antenna theory, and has enabled greater understanding of other antenna types such as the Yagi, or even the traveling wave.

\section{References}

[1] Krauss, J. 1950. Antennas. New York: McGraw-Hill, pp. 11-37.

[2] Stutzman, W., and Thiele, G. 2012. Antenna Theory and Design, 3ed. Hoboken, NJ: John Wiley\&Sons, pp. 23-205.

[3] Ulaby, F., Michielssen, E., and Ravaioli, U. 2010. Fundamentals of Applied Electromagnetics, $6 E$. Englewood Cliffs: Prentice-Hall, pp. 402-448.

[4] Balani, J. 2005. Antenna Theory Analysis and Design, 3E. Hoboken, NJ: John Wiley \& Sons, pp. 1-184.

[5] Silver, H., ed. 2011. The ARRL Antenna Book, $22^{\text {nd }}$ ed. Section 2: Dipole and Monopoles. Newington, CT American Radio Relay League. 